\title{
MASSIVE YOUNG CLUSTERS
}

\author{
Jesús Maíz-Apellániz ${ }^{1,2}$ \\ 1 Space Telescope Science Institute, Baltimore, USA \\ 2 Space Telescope Division, ESA, ESTEC, Noordwijk, Netherlands
}

\begin{abstract}
In the last decade we have come to realize that the traditional classification of stellar clusters into open and globular clusters cannot be easily extended beyond the realm of the Milky Way, and that even for our Galaxy it is not fully valid. The main failure of the traditional classification is the existence of Massive Young Clusters (MYCs), which are massive like Globular Clusters (GCs) but also young like open clusters. We describe here the mass and age distributions of clusters in general with an emphasis on MYCs. We also discuss the issue of what constitutes a cluster and try to establish a general classification scheme.
\end{abstract}

\section{Introduction}

The traditional classification for Milky Way stellar clusters is that they are either globular or open. Globular clusters are old $(\sim 10 \mathrm{Ga})$, massive $\left(3 \cdot 10^{4}-3 \cdot 10^{6} \mathrm{M}_{\odot}\right)$, metal-poor, and spherically-symmetric members of the Galactic halo. Open clusters are young $(\lesssim 1 \mathrm{Ga})$, low-mass $\left(<5 \cdot 10^{3}\right.$ $\left.\mathrm{M}_{\odot}\right)$, metal-rich, and non-spherically-symmetric members of the Galactic disk. The increase in resolution and light-gathering power provided by HST and the new generation of ground-based telescopes has taught us that such classification is not valid for other galaxies and that even for the Milky Way it is not completely correct. Some clusters can be both young and massive and some galaxies can have large numbers of such Massive Young Clusters (or MYCs, Maíz-Apellániz, 2001; Whitmore et al., 1999; Larsen and Richtler, 1999), which are the object of this review. This paper is divided into three sections, each one of them corresponding to the three words that make up the name of these objects: Massive (what are the masses of stellar clusters? how does the mass influence the dynamical evolution of the cluster?), Young (what is the history of stellar cluster formation? what do we know about the 
youngest clusters?), and Clusters (what is the internal structure of a stellar cluster? how do we classify them?).

\section{Massive \\ Mass distributions}

Recent studies have shown that the luminosity distribution of young stellar clusters is a power law $\left(d N / d L_{c l} \propto L_{c l}^{\alpha}\right)$ with $\alpha \approx-2$ over a large range of cluster luminosities (Whitmore et al., 1999; Larsen, 2002). On the other hand, it has been known for some time that GC systems have log-normal luminosity distributions. The mean value of the distribution for our Galaxy is $M_{V}=-7.36 \pm 0.17$ and most other well-studied galaxies have values in the range $M_{V}=-6.9$ to -7.6 (Harris, 1991).

The masses of Galactic globular clusters are well known (at least within a factor of two) since the resolution into individual stars allows for detailed dynamical modeling using radial velocities and/or proper motions (Meylan, 2002). Recent work using HST promises to improve the uncertainties (Anderson and King, 2003). The mass function of the Milky Way thus measured also follows a log-normal distribution (Fall and Zhang, 2001) with its peak centered at $2 \cdot 10^{5} \mathrm{M}_{\odot}$. But what about MYCs, where most of the light is produced by short-lived stars which constitute only a relatively small fraction of the total mass? Do they have masses similar to GCs, implying that they have "normal" stellar IMFs that extend to low masses, or are they deficient in low-mass stars? The masses of MYCs are harder to measure due to inadequate spatial resolution (implying the use of integrated data) and to the fact that the stars which produce the most useful lines for measuring velocity dispersions, red supergiants (Ho and Filippenko, 1996; Smith and Gallagher, 2001), are not present in the earliest stages of MYCs. Also, some clusters have double cores (Maíz-Apellániz, 2001), others are heavily extinguished, and some measurements of the velocity dispersion can be affected by the presence of binaries (Bosch et al., 2001). Results show that most MYCs have indeed normal stellar IMFs and are as massive as GCs (Ho and Filippenko, 1996; Larsen et al., 2001); some clusters may have somewhat anomalous stellar IMFs (Smith and Gallagher, 2001) but the observational problems listed above could be a factor in those cases. Therefore, if the stellar IMF is constant for all or most of the clustermass spectrum, the mass function of young clusters (the cluster IMF) should have the same functional shape as the luminosity function, i.e. a power law with $\alpha \approx-2$. 


\section{Cluster evolution and survival}

Several processes drive the dynamical evolution of a cluster: mass loss due to stellar winds and SNe, a process that heats the cluster; two-body interactions that lead to relaxation, energy redistribution as a function of stellar mass, stellar ejections, evaporation, and possible core-collapse; binary star formation, another cluster-heating process; and tidal interactions in the form of static tidal fields, tidal shocks, and dynamical friction (all leading towards a faster destruction of the cluster) and tidal merging (Gerhard, 2000). These processes are known to be tearing apart GCs little by little (Rockosi et al., 2002).

How will these processes affect the evolution of MYCs. Will they become the GCs of the future? Fall and Rees, 1977 determined that in order for a cluster to survive for a Hubble time, a large initial mass $(\approx$ $3 \cdot 10^{4} \mathrm{M}_{\odot}$ ) was required. For lower-mass clusters the combined effect of two-body interactions and tidal forces was too strong for the cluster to last that long. Since we have seen that MYCs are indeed as massive as GCs, this condition for long-term survival appears to be satisfied. However, why is it that the mass function for young clusters is so different to the one of GCs? The answer comes from the numerical simulations of Fall and Zhang, 2001: since the above mentioned processes destroy low-mass clusters more efficiently than high-mass ones and since even the latter lose stars little by little, an initial power-law mass function is easily converted into a quasi-log-normal one (actually, almost any reasonable initial mass function is converted into a quasi-log-normal one). Boutloukos and Lamers, 2003 backed some of these conclusions with observations of 4 galaxies where the data can be explained if low-mass clusters are preferentially destructed. Furthermore, those authors observe that survival time scales are a function of the environment, as expected: the central regions of spiral galaxies, such as M51 and M33, destroy clusters faster than in the solar neighborhood, located at a larger galactocentric distance. A dwarf irregular galaxy such as the SMC has an even more benign environment, due to the weakness of tidal effects there. Therefore, a $10^{4} \mathrm{M}_{\odot}$ is expected to last less than $100 \mathrm{Ma}$ in the inner regions of M51 but could last close to a Hubble time in the SMC.

\section{Young History and triggering}

Star cluster (of any mass) formation is a continuous process, as the Galactic open cluster population shows, peppered with occasional bursts (Boutloukos and Lamers, 2003). The formation history of massive star 
clusters is harder to study due to their relative scarcity for young ages and to the difficulties associated with measuring ages for unresolved systems (Kissler-Patig, 2002). GC systems show a color bimodality that can be interpreted as the result of two bursts of star formation but also as the merger (without new cluster formation) of two preexisting galaxies; the latter model has some problems that make it less likely ( Kundu and Whitmore, 2002). The observed new cluster population in current mergers such as the Antennae indicate that this process is quite effective in forming considerable numbers of MYCs (Whitmore et al., 1999).

MYCs appear to require galactic-scale massive events (such as galaxy formation or mergers) to form in large quantities but more modest numbers can be produced without resorting to such processes. Thus, we have dwarf starbursts such as NGC 4214, a Magellanic irregular where a few MYCs have formed along the galactic bar in the last $\sim 10 \mathrm{Ma}$ (MacKenty et al., 2000). Even in the Local Group, where no major mergers have taken place recently, we have two good examples of MYC with ages < $10 \mathrm{Ma}, 30$ Doradus in the LMC and NGC 604 in M33, plus a few other cases (Maíz-Apellániz, 2004). A number of processes, such as gravitational instabilities (caused by e.g. bars), massive-cloud collisions, and galactic tidal interactions, appear to be able to produce massive star clusters (Terlevich, 1997). The relative importance of each process has not been studied in detail yet. Another aspect that probably deserves attention is an in-depth analysis of the cluster IMF: is it identical for low-scale cluster formation processes and for massive ones (the differences in the number of MYCs originating only in a lower number for small-scale events due to the stochastic filling of the cluster IMF) or are there intrinsic differences?

\section{Generations and the surrounding medium}

A compact low-mass cluster is born rather quickly (few $10^{5}$ years, Bonnell et al., 2003) and the core of a MYC is likely to form on similar time scales. Therefore, MYC cores should be well approximated by single-age populations. However, as we will see in the next section, many MYCs have complex structures outside their cores which can be made up of several stellar generations. In 30 Doradus, the best studied MYC, five populations of different ages can be identified (Walborn and Blades, 1997; Grebel and Chu, 2000), from a 20-25 Ma subcluster to an ongoing new generation; the core itself, R136, is 2-3 Ma old. Some of those populations could actually be unrelated to the cluster (i.e. they could be in the vicinity but not physically associated with it) but there 
is good evidence that others are indeed part of the same cluster. Indeed, other MYCs also have a second generation $\sim 3$ Ma older then the main star formation episode (Parker et al., 1992; Walborn and Parker, 1992).

The analysis of well-resolved MYCs shows that this presence of multiple generations is related to the interaction between the cluster and the surrounding medium (Maíz-Apellániz, 2004 and references therein). Massive Young Clusters are born from Giant Molecular Clouds and the ultraviolet radiation from the massive stars with some help from stellar winds carves an initial cavity in the molecular gas of a few tens of pc in size in the first $\approx 3 \mathrm{Ma}$ by first dissociating and later ionizing the molecular gas. The Giant H II Region around it is formed as a highly-stratified, thin $(1-2$ pc) region on the surface of the Giant Molecular Cloud directly exposed to the UV radiation that can extend for several tens of pc and that, in many senses, is nothing but a scaled-up version of what we observe in lower-mass nearby H II regions (Scowen et al., 1998; Ferland, 2001). This process also drives shock waves into the molecular cloud, compressing the gas and triggering the birth of new stars. After $\approx 3 \mathrm{Ma}$, the first $\mathrm{SNe}$ start exploding and the resulting shock waves enhance the formation of this second generation. However, they also contribute to sweeping away the molecular gas, so the formation of new stars eventually stops shortly thereafter.

\section{Clusters}

\section{SSCs, SOBAs, cores, and halos}

Many stars are not formed in isolation but rather they are born in groups. Sometimes, the group is compact enough to be bound, at least for a period much longer than a typical orbital time for a given star, and we have a (real) cluster. In other occasions, the group is too extended and, although the stars are born with similar velocity vectors (which differentiates them from nearby non-group members), the tidal field of the galaxy easily disrupts the group within one galactic rotation. In that case, the group is called an (OB) association. It is not uncommon to have clusters (bound groups) within more extended associations, with both originating from the same progenitor molecular cloud (de Zeeuw et al., 1999). This description was originally derived from the study of the relatively young low-mass clusters and associations in the solar neighborhood. In the past decade we have found out that it can be easily extended to the upper end of the young cluster mass spectrum. Thus, MYCs can be divided into two types: Super Star Clusters (SSCs) are organized around a compact (half-light radius, $r_{1 / 2}=1-3 \mathrm{pc}$ ) core while Scaled OB Associations (SOBAs) lack such structure and are 


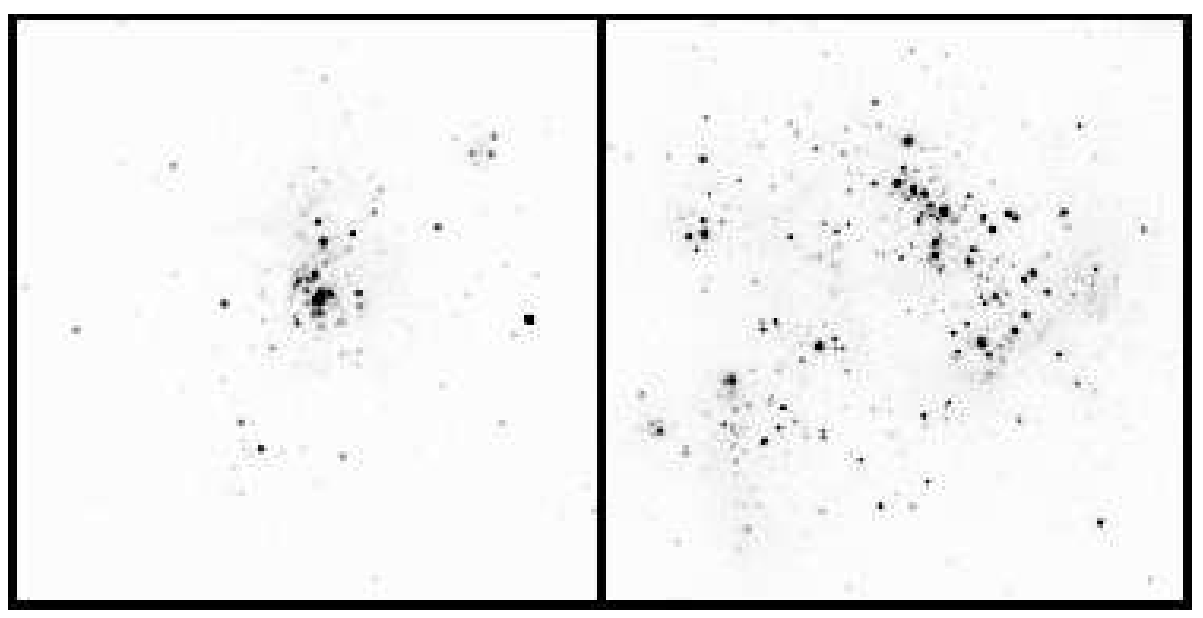

Figure 1. A comparison between 30 Doradus, an SSC with a halo (left), and NGC 604 , a SOBA (right). Both images were obtained using similar filters (continuum at $\mathrm{H} \beta$ for 30 Doradus, WFPC2 F547M for NGC 604), and have the same physical size $(120 \mathrm{pc} \times 120 \mathrm{pc})$, orientation $(\mathrm{N}$ at top), and resolution (the ground-based 30 Doradus image was degraded to attain this objective). R136, the SSC core, is the bright object at the center of the 30 Doradus image.

more extended objects, with $r_{1 / 2}>10$ pc (Hunter, 1995; Maíz-Apellániz, 2001). SSCs are bound objects and represent the high mass end of young stellar clusters while SOBAs are (at least from a global point of view) unbound and are the massive relatives of regular $\mathrm{OB}$ associations ${ }^{1}$. Furthermore, the core of some SSCs is surrounded by extended halos which are themselves similar to SOBAs in terms of structure and number of stars, thus representing the high-mass equivalent of those associations with clusters inside. All three types of MYCs (SSCs with and without halos, and SOBAs) are well represented in the sample available in nearby galaxies (Maíz-Apellániz, 2001).

Why do MYCs come in these varieties? Part of the solution was discussed in the previous section: the influence of an SSC in its surrounding medium can produce, if sufficient material is available, a second generation of stars which would form (part of) the halo. A more comprehensive explanation comes from recent numerical simulations of galactic disks which show that molecular clouds are high-density, high-pressure

\footnotetext{
${ }^{1}$ Note that by calling a SOBA a MYC we are introducing a slight terminological inconsistency with respect to the low-mass end of the spectrum, since the classical definition of a stellar cluster implies a bound object. Here we are applying a less restrictive definition of cluster to include associations and SOBAs, i.e. to mean a group of stars born from the same molecular cloud within a short $(\sim 10 \mathrm{Ma})$ period of time.
} 
regions that form mainly by turbulent ram pressure (as opposed to by self-gravity), which has its ultimate origin mostly in SN explosions ( Mac Low, 2004). In this scenario, molecular clouds are transient features which are easily created and destroyed. Gravity would play a role only after turbulent pressure creates filamentary structures dense enough to start collapsing (Bate et al., 2003). The simulations in those references are for "normal" conditions (leading to low-mass cluster formation) but the similarities that we have found between the structural properties of low-mass and high-mass clusters and associations suggest that MYCs may form in the same way, a hypothesis also supported by the hierarchical nature of cluster formation (Bonnell et al., 2003). The most important difference between high-mass and low-mass clusters would be the need for a large initial amount of gas and for an extremely high pressure in order to compress it into a relatively small volume; such conditions could be caused by any of the mechanisms (galactic collisions, gravitational instabilities...) discussed in the previous section. The hierarchical nature of the process suggests that the same type of filamentary structures should form in the dense molecular gas during the early stages of formation of a MYC. Subclusters would then form along those structures and, if a region is dense enough to produce a large number of them within a small volume, a core would be formed when they merge. The rest of the subclusters would form the halo or SOBA part of the MYC, with the possible help of the shock waves created by nearby stars as described in the previous section. In a time scale of the order of 10-30 Ma (the typical orbital periods around the center of the cluster for stars located at radii of $10-20 \mathrm{pc}$ ) the relative positions of the stars there would bear little resemblance to their original ones, but, for clusters younger than that, the halo could still have some memory of the original filamentary structure of the molecular gas. Indeed, some observations support this idea (Maíz-Apellániz, 2001; Maíz-Apellániz, 2004). Given the size of several tens of pc of the cloud, the whole MYC formation process could take $\sim 10$ Ma from the time when the first stars are born until the time when the molecular material is dispersed, a value also consistent with the observed properties of well-studied MYCs.

\section{Size does matter: cluster survival and classification}

As already described by Fall and Rees, 1977, a high mass is not the only condition necessary for long-term cluster survival: size does matter. Clusters which are too compact are easily affected by two-body interactions (though their immediate destiny is probably not destruction but only expansion) while clusters that are too extended get disrupted by 
tides, as we have already mentioned. SSCs have the right intermediate size to ensure survival (Maíz-Apellániz, 2001) and one would expect them to become GCs in the future. SOBAs, however, are too extended to survive for a long period of time and are expected to dissolve rather easily and its members should become part of the non-cluster population of their host galaxy. Intense star formation episodes (i.e. starbursts $^{2}$ ) can produce either SSCs or SOBAs or both, so one should expect them to enrich not only the massive cluster population of their host galaxies but also their field stellar population as a result of the dissolution of the SOBAs.

What does all of this tell us with respect to the classification of stellar groups? First, that the most clear division is not between open and globular clusters but between real clusters and associations, both of which are the children of Giant Molecular Clouds, the first being bound and the second unbound objects. Second, that real clusters only survive for a long time if they are massive enough. Third, that SSCs are likely to become GCs after some time. Indeed, it is probably a good idea to define an SSC as a cluster which has the right size and enough mass to become a $\mathrm{GC}$ in the future. With those ideas in mind, I propose the following classification scheme:

\begin{tabular}{ccccc} 
& \multicolumn{2}{c}{ Compact (bound) } & \multicolumn{2}{c}{ Extended (unbound) } \\
Young & Low mass & High mass & Low mass & High mass \\
\cline { 2 - 5 } Old & Open & SSC & OB association & SOBA \\
\cline { 3 - 5 } & & Globular & & \\
\cline { 3 - 5 } & & &
\end{tabular}

Support for this work was provided by NASA through grant GO09096.01-A from the Space Telescope Science Institute, Inc., under NASA contract NAS5-26555, and by the Spanish Government grant AYA-20013939 .

\section{References}

Anderson, J. and King, I. R. (2003). AJ, 126:772-777.

Bate, M. R., Bonnell, I. A., and Bromm, V. (2003). MNRAS, 339:577-599.

Bonnell, I. A., Bate, M. R., and Vine, S. G. (2003). MNRAS, 343:413-418.

Bosch, G., Selman, F., Melnick, J., and Terlevich, R. (2001). A\&A, 380:137-141.

Boutloukos, S. G. and Lamers, H. J. G. L. M. (2003). MNRAS, 338:717-732.

de Zeeuw, P. T. et al. (1999). AJ, 117:354-399.

Fall, S. M. and Rees, M. J. (1977). MNRAS, 181:37P-42P.

Fall, S. M. and Zhang, Q. (2001). ApJ, 561:751-765.

${ }^{2}$ Note that this term is used sometimes to denote only episodes of very high intensity and of galactic proportions. 
Ferland, G. J. (2001). PASP, 113:41-48.

Gerhard, O. (2000). In Massive Stellar Clusters, A. Lançon and. C. M. Boily (eds.), ASP Conf. Ser. 211 (San Francisco: ASP), pages 12-24.

Grebel, E. K. and Chu, Y. (2000). AJ, 119:787-799.

Harris, W. E. (1991). ARA $\& A, 29: 543-579$.

Ho, L. C. and Filippenko, A. V. (1996). ApJ, 472:600-610.

Hunter, D. A. (1995). Rev. Mex. Astron. Astrofís. (conference series), 3:1-7.

Kissler-Patig, M. (2002). In Extragalactic Star Clusters, E. Grebel, D. Geisler, and D. Minniti (eds.), Proc. IAU Symposium No. 207 (San Francisco: ASP), pages $207-217$.

Kundu, A. and Whitmore, B. (2002). In Extragalactic Star Clusters, E. Grebel, D. Geisler, and D. Minniti (eds.), Proc. IAU Symposium No. 207 (San Francisco: $A S P)$, pages $229-237$.

Larsen, S. S. (2002). AJ, 124:1393-1409.

Larsen, S. S. et al. (2001). ApJ, 556:801-812.

Larsen, S. S. and Richtler, T. (1999). A\&A A, 345:59-72.

Mac Low, M.-M. (2004). These proceedings.

MacKenty, J. W. et al. (2000). AJ, 120:3007.

Maíz-Apellániz, J. (2001). ApJ, 563:151-162.

Maíz-Apellániz, J. (2004). In The Local Group as an Astrophysical Laboratory, proceedings of a workshop at STScI, Baltimore, 5-8 May 2003, (Cambridge: CUP), in press.

Meylan, G. (2002). In Extragalactic Star Clusters, E. Grebel, D. Geisler, and D. Minniti (eds.), Proc. IAU Symposium No. 207 (San Francisco: ASP), pages 555565.

Parker, J. Wm., Garmany, C. D., Massey, P., and Walborn, N. R. (1992). AJ, 103:12051233.

Rockosi, C. M. et al. (2002). AJ, 124:349-363.

Scowen, P. A. et al. (1998). AJ, 116:163-179.

Smith, L. J. and Gallagher, J. S. (2001). MNRAS, 326:1027-1040.

Terlevich, E. (1997). In Starburst Activity in Galaxies, J. Franco, R. Terlevich and A. Serrano (eds.), Rev. Mex. Astron. Astrofis. (conference series) Vol. 6, pages 243-245.

Walborn, N. R. and Blades, J. C. (1997). ApJS, 112:457-485.

Walborn, N. R. and Parker, J. Wm. (1992). ApJ, 399:L87-L89.

Whitmore, B. C. et al. (1999). AJ, 118:1551. 